\title{
Trastorno de ansiedad en situación de pandemia por COVID-19
}

\section{Anxiety disorder in a COVID-19 pandemic situation}

\author{
Jenifer Isabel Chacón Figueroa \\ Universidad de San Carlos de Guatemala \\ https://orcid.org/0000-0001-8528-9322 \\ jeniferisabelchacon@gmail.com
}

Recibido: $14 / 07 / 2021$

Aceptado: 01/09/2021

\section{Referencia del artículo}

Chacón Figueroa, J. I. (2021). Trastorno de ansiedad en situación de pandemia por COVID-19. Revista Diversidad Científica, 1(1). 169-176.

DOI: https://doi.org/10.36314/diversidad.v1i1.18

\section{Resumen}

OBJETIVO: determinar si existe el trastorno de ansiedad, medido a través del inventario de Beck en el municipio de Zacapa. MÉTODO: estudio descriptivo prospectivo, la recolección de información, se realizó mediante una encuesta distribuida a través de Facebook y WhatsApp. La muestra fue de 380 encuestados. RESULTADOS: de los 380 encuestados en el municipio de Zacapa, el 35.5\% presentaron características clínicas de ansiedad moderada, $25.8 \%$ leve, $20.5 \%$ mínima y el $18.2 \%$ presentaron ansiedad grave. Según la edad, la ansiedad moderada predomina en la población de 42 a 51 años con $48.7 \%$; la ansiedad grave predomina en el grupo etario de 52 a 61 años con el $27.0 \%$. De acuerdo al sexo, la ansiedad moderada predomina en el sexo masculino con $36.3 \%$, seguido, el sexo femenino con $35.0 \%$. Un $47.6 \%$ con ansiedad moderada presentaron una disminución económica del $75 \%$. CONCLUSIÓN: prevalece el grado de ansiedad moderada, que ocupa el 35.5\%. El grupo etario más afectado es la población de 42 a 51 años debido a que están expuestos a mayor carga de responsabilidades familiares, laborales y económicas.

Palabras clave: trastorno de ansiedad, pandemia COVID-19, medidas de higiene y restricción social 


\begin{abstract}
OBJECTIVE: to determine if there is an anxiety disorder, measured through the Beck inventory in the municipality of Zacapa. METHOD: prospective descriptive study, the collection of information was carried out through a survey distributed through Facebook and WhatsApp. The sample was 380 respondents. RESULTS: of the 380 respondents in the municipality of Zacapa, $35.5 \%$ presented clinical characteristics of moderate anxiety, $25.8 \%$ mild, $20.5 \%$ minimal and $18.2 \%$ presented severe anxiety. According to age, moderate anxiety predominates in the population aged 42 to 51 years with $48.7 \%$; severe anxiety predominates in the age group from 52 to 61 years with $27.0 \%$. According to sex, moderate anxiety predominates in males with $36.3 \%$, followed by females with $35.0 \%$. $47.6 \%$ with moderate anxiety presented an economic decrease of $75 \%$. CONCLUSION: the degree of moderate anxiety prevails, occupying $35.5 \%$. The age group most affected is the population between 42 and 51 years of age because they are exposed to a greater burden of family, work and economic responsibilities.
\end{abstract}

Keywords: anxiety disorder, COVID-19 pandemic, hygiene measures and social restriction 


\section{Introducción}

Los trastornos mentales son considerados la principal causa de afección física y mental de tipo emocional, conducta y aprendizaje de un individuo, sin discriminar edad, sexo, condición socioeconómica, cultural y religiosa. Lo cual dificulta a la persona a afrontar las tensiones de la vida cotidiana, y ser incapaz de hacer una contribución a la comunidad (OPS 2018).

En Guatemala, uno de cada cuatro guatemaltecos mayores de 18 años de edad, ha manifestado al menos un trastorno mental; solamente el $2.3 \%$ de la población guatemalteca ha consultado con algún profesional en salud por emociones o problemas de salud mental. En términos generales los trastornos de ansiedad constituyen el grupo de trastornos más frecuente que afecta a la población guatemalteca (OPS/ OMS 2012).

A principios del año 2020, las condiciones sociales, económicas y culturales sufrieron fuertes impactos a nivel mundial, debido al virus COVID-19. El estado de Guatemala, a través del ministerio de Salud y demás instancias gubernamentales, han implementado medidas no farmacológicas para la mitigación y control de la pandemia, éstas son modificadas conforme al avance de la enfermedad. Las medidas de higiene y restricciones sociales implementadas al momento de realizar el estudio son: distanciamiento social, uso obligatorio de mascarilla, confinamiento, toque de queda, desinfección en lugares públicos y privados, cuarentena, cancelación de reuniones culturales y religiosas, cierre de negocios, entre otras. Éstas, son sin duda, situaciones que pueden afectar la salud mental de la población.

El estudio se realizó con una muestra de 380 personas, mediante una distribución de los encuestados en 4 grupos etarios. Para la recolección de datos, fue utilizada la herramienta del inventario de Beck para ansiedad. El proceso de investigación, se llevó acabo de manera totalmente virtual, mediante la plataforma de WhatsApp y Facebook.

Los resultados arrojan que, en la población del municipio de Zacapa, predomina la ansiedad moderada en un $35.5 \%$ (135), el grupo etario más afectado por este tipo de ansiedad es de $42-51$ años, con un $48.7 \%$ (38). Con una diferencia poco marcada, los hombres presentaron mayor porcentaje de ansiedad que las mujeres. Los grupos de estudio con mayor prevalencia en ansiedad de moderada a grave se inclinaron en las categorías en donde la economía, la salud, la responsabilidad social y cultural llevan consigo una relación importante. 


\section{Contenido}

Se realizó un estudio descriptivo prospectivo, con 380 personas de 22 a 61 años de edad, subdividido en 4 grupos etarios, por intervalos de 10 años en el municipio de Zacapa. Se utilizó la técnica de encuesta virtual mediante dispositivos electrónicos (teléfonos, tabletas y computadoras), el cual, se distribuyó un link que contenía la información donde explicaba la finalidad de la investigación, e indicaba la participación de forma voluntaria.

El formulario se dividió en dos grandes aspectos; datos generales y datos propios del inventario de ansiedad de Beck y se almacenó a través de la plataforma virtual, luego se procedió a la descarga y tabulación en una hoja de cálculo de Microsoft Excel.

El inventario de Beck (BAI) se usa para medir la gravedad del nivel de ansiedad en un individuo. Está compuesta por una serie de 21 ítems que evalúan los síntomas de ansiedad como el sudor, aceleración del ritmo cardiaco, irritabilidad y falta de aliento. los resultados del instrumento indican el nivel de ansiedad de las personas evaluadas, las puntuaciones establecidas son: 0-7 ansiedad mínima, 8-15 ansiedad leve, 16-25 ansiedad moderada, 26-63 ansiedad grave.

De los 380 encuestados en el municipio de Zacapa, el 35.5\% (135) presentaron características clínicas principalmente de ansiedad moderada, $25.8 \%$ (98) ansiedad leve, $20.5 \%$ (78) ansiedad mínima y el $18.2 \%$ (69) presentaron ansiedad grave.

Según el grupo etario, la ansiedad moderada predomina en la población de 42 a 51 años con $48.7 \%$ (38); la ansiedad grave predomina en el grupo etario de 52 a 61 años con el $27.0 \%$ (17).

De acuerdo al sexo, la ansiedad moderada predomina en el sexo masculino con $36.3 \%$ (58), seguido, el sexo femenino con $35.0 \%$ (77). Un $47.6 \%$ (30) con ansiedad moderada presentaron una disminución económica del $75 \%$. 
Figura No.1 Distribución de las personas que presentan grados de ansiedad, clasificados a través del Inventario de Beck.

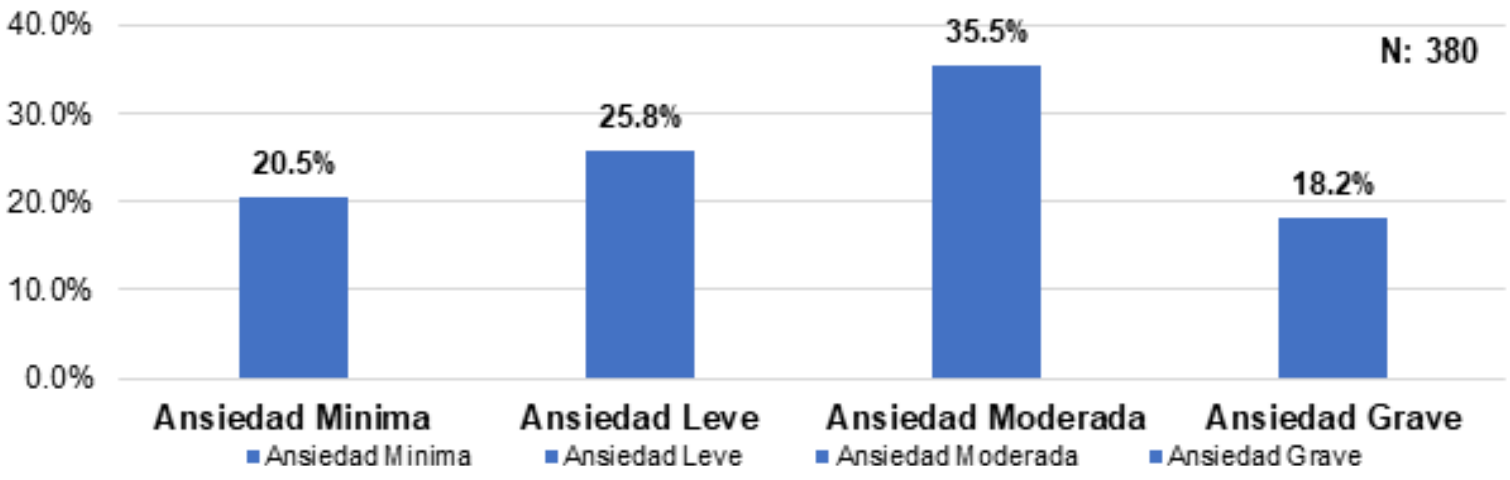

Fuente: recolección de datos, 2020.

De las 380 personas encuestadas el 35.5\% (135) presentaron predominio en características clínicas de trastornos de ansiedad moderada, un $25.8 \%(98)$ ansiedad leve, un $20.5 \%$ (78) ansiedad mínima y con una minoría del $18.2 \%$ (69) presentaron ansiedad grave.

Figura No. 2 Distribución de los grados de ansiedad según sexo, identificados por el Inventario de Beck.

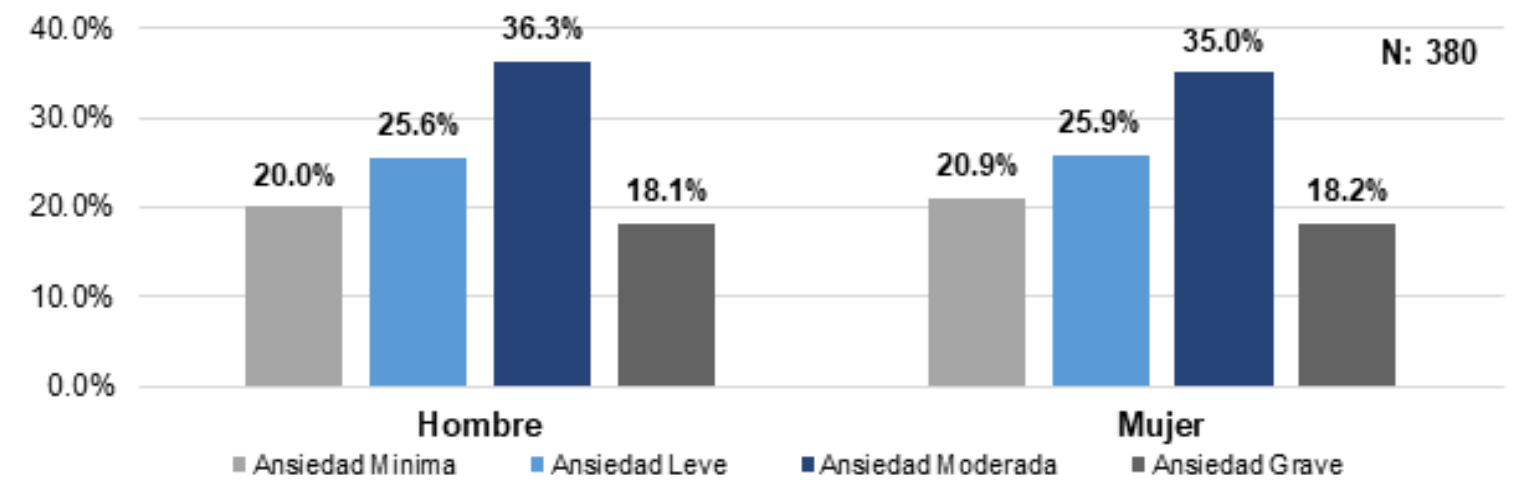

Fuente: recolección de datos, 2020.

En relación al sexo, se observa que la característica de trastorno de ansiedad moderada predomina en los hombres, con el $36.3 \%$ (58), seguido por el sexo femenino con el $35.0 \%$ (77) con ansiedad moderada, sin embargo, el trastorno de ansiedad grave, se presenta en mayor porcentaje en el grupo de mujeres con un $18.2 \%$ (40). 
Figura No. 3 Distribución de los grados de ansiedad según grupo etario, identificados por el Inventario de Beck.

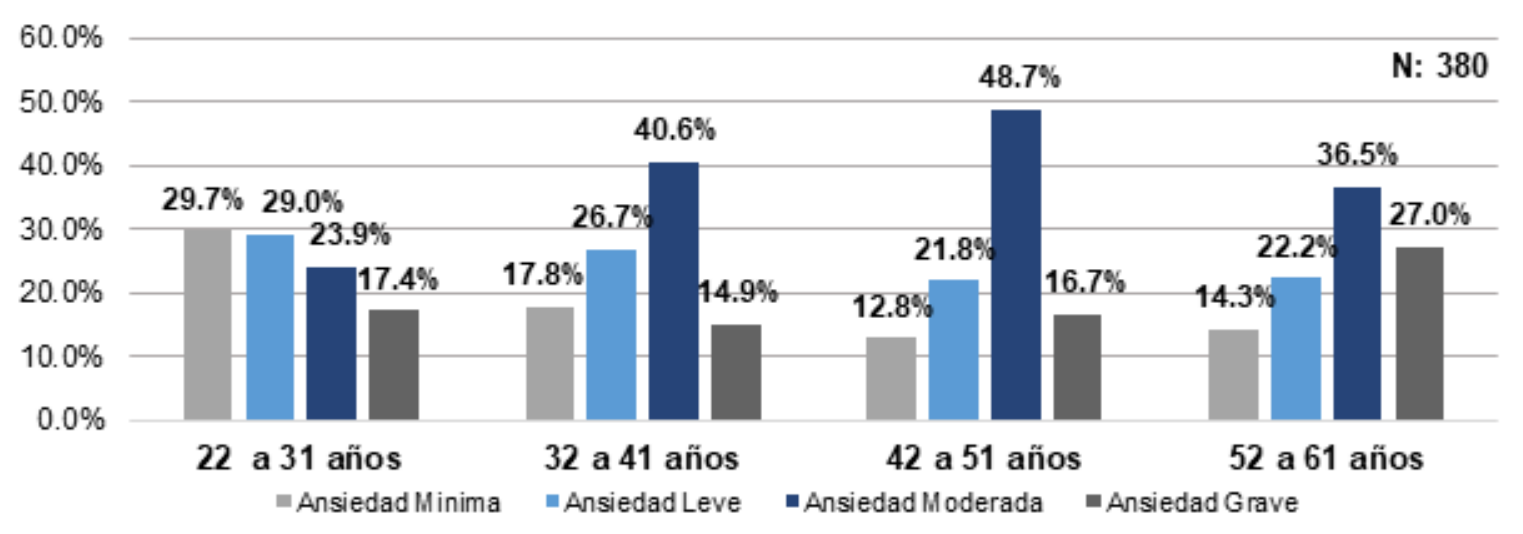

Fuente: recolección de datos, 2020.

En el análisis se observa que el grupo etario de 42-51 presentaron síntomas predominantes de trastornos de ansiedad moderada el $48.7 \%$ (38), sin embargo, el grupo etario de $52-61$ se encuentra de una forma predominante del $27.0 \%$ (17) con ansiedad grave, el rango de $22-31$ presentan alto porcentaje con $29.7 \%$ (41) de ansiedad mínima.

\section{Discusión}

Se determino que, en la población del municipio de Zacapa, de las 380 personas encuestadas el $35.5 \%$ (135) presentaron predominio en características clínicas de trastornos de ansiedad moderada, un 25.8\% (98) ansiedad leve, un 20.5\% (78) ansiedad mínima y con una minoría del 18.2\% (69) presentaron ansiedad grave.

Con relación al sexo, se observa que la característica de trastorno de ansiedad moderada predomina en los hombres, con el $36.3 \%$ (58), seguido por el sexo femenino con el $35.0 \%$ (77) con ansiedad moderada, sin embargo, el trastorno de ansiedad grave, se presenta en mayor porcentaje en el grupo de mujeres con un $18.2 \%(40)$.

Con relación al grupo etario de 42-51 presentaron síntomas predominantes de trastornos de ansiedad moderada el $48.7 \%$ (38), sin embargo, el grupo etario de 52-61 se encuentra de una forma predominante del $27.0 \%$ (17) con ansiedad grave, el rango de 22-31 presentan alto porcentaje con $29.7 \%$ (41) de ansiedad mínima. 
Sin embargo, se determinó que los que tuvieron "disminución económica del 100\%", presentaron $36.8 \%$ (7) ansiedad grave. La categoría de "disminución económica del $75 \%$ " presentaron $47.6 \%$ (30) ansiedad moderada. La muestra con "disminución económica del $50 \%$ " presentaron $25 \%$ (20) ansiedad mínima y las personas "sin disminución económica" 40.4\% (42) presentaron ansiedad leve.

\section{Declaración}

El estudio se realizó con fines médicos, de acuerdo a las directrices de la Declaración de Helsinki y Código de Ética y Buenas Prácticas COPE, con el consentimiento de las personas que intervinieron en el estudio.

\section{Conflicto de intereses}

La autora declara no tener ningún conflicto de intereses.

\section{Referencias}

OPS (Organización Panamericana de la Salud). 2018. La carga de los trastornos mentales en la Región de las Américas, 2018 (en línea). Washington, Estados Unidos de América, OPS/OMS. 50 p. Consultado 19 may. 2020. Disponible en https://iris.paho.org/bitstream/handle/10665.2/49578/9789275320280_spa. pdf? sequence $=9$ \&isAllowed $=y$.

OPS (Organización Panamericana de la Salud) /OMS (Organización Mundial de la Salud). 2012. Día mundial de la salud mental: la depresión, una crisis global (en línea, sitio web). Guatemala. Consultado 5 jun. 2020. Disponible en https:// www.paho.org/gut/index.php?option=com_content\&view=article\&id=610: dia-mundial-de-salud-mental-depresion-una-crisis-global\&ltemid=405.

\section{Sobre la autora}

\section{Jenifer Isabel Chacón Figueroa}

Es graduada en la Carrera Médico y Cirujano de la Universidad de San Carlos de Guatemala. 
Copyright (c) Jenifer Isabel Chacón Figueroa

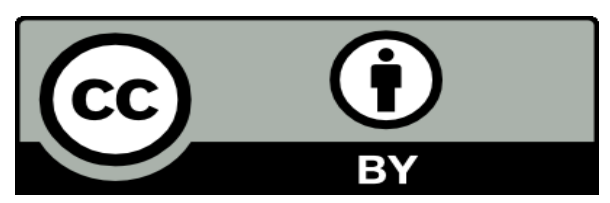

Este texto está protegido por una licencia CreativeCommons 4.0.

Usted es libre para compartir, copiar y redistribuir el material en cualquier medio o formato y adaptar el documento, remezclar, transformar y crear a partir del material para cualquier propósito, incluso comercialmente, siempre que cumpla la condición de atribución: usted debe reconocer el crédito de una obra de manera adecuada, proporcionar un enlace a la licencia, e indicar si se han realizado cambios. Puede hacerlo en cualquier forma razonable, pero no de forma tal que sugiera que tiene el apoyo del licenciante o lo recibe por el uso que hace. 